Parametric characterization of the spatial structure of partially coherent and partially polarized beams

This article has been downloaded from IOPscience. Please scroll down to see the full text article.

2004 J. Opt. A: Pure Appl. Opt. 6 S67

(http://iopscience.iop.org/1464-4258/6/3/012)

View the table of contents for this issue, or go to the journal homepage for more

Download details:

IP Address: 147.96.14.16

The article was downloaded on 11/06/2013 at 17:57

Please note that terms and conditions apply. 


\title{
Parametric characterization of the spatial structure of partially coherent and partially polarized beams
}

\author{
R Martínez-Herrero, G Piquero and P M Mejías \\ Departamento de Óptica, Facultad de Ciencias Físicas, Universidad Complutense de Madrid, \\ 28040 Madrid, Spain
}

Received 11 September 2003, accepted for publication 9 January 2004

Published 24 February 2004

Online at stacks.iop.org/JOptA/6/S67 (DOI: 10.1088/1464-4258/6/3/012)

\begin{abstract}
On the basis of the second-order intensity moments formalism, the relationship between the spatial structure and the overall polarization characteristics of partially polarized Gaussian Schell-model beams of a certain kind has been investigated. More specifically, attention has been focused on a type of source that cannot be distinguished from ordinary Gaussian Schell-model fields when polarization measurements are disregarded. For this class of beams several general properties have been obtained that enable us to link the beam coherence polarization matrix and the beam quality parameter with certain polarization degrees recently introduced in the literature.
\end{abstract}

Keywords: polarization, coherence, beam quality

\section{Introduction}

The characterization of the spatial behaviour of laser beams has been a topic of interest in the past ten years [1-12]. Attention had mainly been focused on scalar treatments assuming totally and uniformly polarized beams. Realistic beams should, however, be considered as partially coherent and partially polarized fields and, in general, they can exhibit non-uniform distributions of the polarization state across their transverse profiles. Accordingly, for a complete description of a general beam it would be necessary to handle both spatial and polarization characteristics. So far the focusing and divergence capabilities of a light beam have been represented by the so-called beam quality parameter, extensively used in scalar treatment [7-12] and whose simple generalization to the vectorial case has been reported elsewhere [13]. The characterization of the polarization structure is, however, a more involved problem, and several proposals based on complementary overall polarization degrees have recently been published [14-17].

But a problem arises concerning the relationship between the spatial beam features and the polarization behaviour upon propagation. There is no general answer to this question. Note that in this sense a number of beams can be given with the same value of the quality parameter but having very different polarization distributions.
We have, however, analysed this problem for a certain class of partially polarized Gaussian Schell-model (PGSM) beams recently described in the literature [18, 19]. More specifically, in the present work simple analytical expressions are obtained that relate the spatial beam characteristics and the degrees of polarization of such fields.

This paper is arranged as follows. In the next section we introduce the main definitions and the key parameters to be used. The relationships between the overall spatial characteristics and the polarization parameters are derived in section 3 . Finally, the main conclusions are listed in section 4 .

\section{Definitions and key beam parameters}

As is well known, within the paraxial approximation any partially coherent and partially polarized quasimonochromatic beam propagating along the $z$-axis can be described at some transverse plane by the so-called beam coherence polarization $(\mathrm{BCP})$ matrix $[20,21]$ :

$$
\hat{J}\left(\mathbf{r}_{1}, \mathbf{r}_{2}, z\right)=\left(\begin{array}{cc}
J_{\mathrm{ss}}\left(\mathbf{r}_{1}, \mathbf{r}_{2}, z\right) & J_{\mathrm{sp}}\left(\mathbf{r}_{1}, \mathbf{r}_{2}, z\right) \\
J_{\mathrm{ps}}\left(\mathbf{r}_{1}, \mathbf{r}_{2}, z\right) & J_{\mathrm{pp}}\left(\mathbf{r}_{1}, \mathbf{r}_{2}, z\right)
\end{array}\right),
$$

where

$$
\begin{aligned}
& J_{\alpha \beta}\left(\mathbf{r}_{1}, \mathbf{r}_{2}, z\right)=\left\langle E_{\alpha}^{*}\left(\mathbf{r}_{1}, z ; t\right) E_{\beta}\left(\mathbf{r}_{2}, z ; t\right)\right\rangle \\
& (\alpha, \beta=\mathrm{s}, \mathrm{p}) .
\end{aligned}
$$


Here, the asterisk symbolizes the complex conjugate, $\mathbf{r}_{1}$ and $\mathbf{r}_{2}$ are position vectors lying in the plane $z=$ constant, the angle brackets denote the time average and $E_{\alpha}(\alpha=\mathrm{s}, \mathrm{p})$ represents a Cartesian component of the time-dependent electric field.

The BCP matrix provides a joint description of the polarization and spatial coherence properties of the beam. In particular, its diagonal elements, $J_{\mathrm{ss}}$ and $J_{\mathrm{pp}}$, can be regarded as the mutual intensities of the beam after crossing ideal linear polarizers whose transmission axes are the s-axis and p-axis, respectively.

The overall spatial characterization of the beam will then be given by the second-order intensity moments of the elements of the BCP matrix, defined by the expression

$$
\begin{gathered}
\langle\alpha \beta\rangle_{i j}=\frac{1}{I_{i j}} \frac{k^{2}}{4 \pi^{2}} \iiint \alpha \beta J_{i j}\left(\mathbf{r}_{1}, \mathbf{r}_{2}, z\right) \\
\times \exp \left[\mathrm{i} k\left(\mathbf{r}_{1}-\mathbf{r}_{2}\right) \cdot \eta\right] \mathrm{d} \mathbf{r}_{1} \mathrm{~d} \mathbf{r}_{2} \mathrm{~d} \boldsymbol{\eta},
\end{gathered}
$$

with

$$
\begin{aligned}
I_{i j}= & \frac{k^{2}}{4 \pi^{2}} \int J_{i j}(\mathbf{r}, \mathbf{r}, z) \mathrm{d} \mathbf{r} ; \\
& i, j=\mathrm{s}, \mathrm{p} ; \quad \alpha, \beta=x, y, u, v,
\end{aligned}
$$

where $k \boldsymbol{\eta}=(k u, k v)=\left(k_{x}, k_{y}\right)$ gives the wavevector components along the Cartesian $x$ - and $y$-axes. Consequently, $u$ and $v$ represent angles of propagation (without taking the evanescent waves into account).

In equation (3) $\langle\alpha \beta\rangle_{i i}, i=\mathrm{s}$, p represent the second-order moments associated with the respective field components, $I_{i i} \equiv I_{i}, i=\mathrm{s}$, p, is the intensity of the $i$-component, and $I=I_{\mathrm{s}}+I_{\mathrm{p}}$ denotes the total intensity. For the sake of simplicity, in what follows it will be assumed that the first-order beam moments, $\langle x\rangle,\langle y\rangle,\langle u\rangle$ and $\langle v\rangle$, equal zero. This is not a true restriction, since it is equivalent to a shift of the Cartesian coordinate system.

Taking this into account, the beam quality parameter at the waist plane reads

$$
Q=\left\langle r^{2}\right\rangle\left\langle\eta^{2}\right\rangle
$$

where the second-order moments $\left\langle r^{2}\right\rangle=\left\langle x^{2}+y^{2}\right\rangle$ and $\left\langle\eta^{2}\right\rangle=$ $\left\langle u^{2}+v^{2}\right\rangle$ now include both field components, that is,

$$
\begin{aligned}
& \left\langle r^{2}\right\rangle=\frac{I_{\mathrm{s}}}{I}\left\langle r^{2}\right\rangle_{\mathrm{s}}+\frac{I_{\mathrm{p}}}{I}\left\langle r^{2}\right\rangle_{\mathrm{p}}, \\
& \left\langle\eta^{2}\right\rangle=\frac{I_{\mathrm{s}}}{I}\left\langle\eta^{2}\right\rangle_{\mathrm{s}}+\frac{I_{\mathrm{p}}}{I}\left\langle\eta^{2}\right\rangle_{\mathrm{p}},
\end{aligned}
$$

with $\left\langle r^{2}\right\rangle_{i} \equiv\left\langle r^{2}\right\rangle_{i i}, i=\mathrm{s}, \mathrm{p}$. The presence of the factors $I_{i} / I$, $i=\mathrm{s}, \mathrm{p}$, in the right-hand side of equations (6) and (7) arises from the different normalization constants, $I$ and $I_{i}$, of the moments associated with the global beam and with each field component, respectively.

In order to characterize the polarization of the beam, several overall measurable parameters have been proposed in the literature:

- Standard degree of polarization $P_{\mathrm{st}}$ :

$$
\begin{aligned}
P_{\mathrm{st}} & =\left\{\left(\left\lceil\int J_{\mathrm{ss}}(\mathbf{r}, \mathbf{r}, z) \mathrm{d} \mathbf{r}-\int J_{\mathrm{pp}}(\mathbf{r}, \mathbf{r}, z) \mathrm{d} \mathbf{r}\right]^{2}\right.\right. \\
& \left.+4\left|\int J_{\mathrm{sp}}(\mathbf{r}, \mathbf{r}, z) \mathrm{d} \mathbf{r}\right|^{2}\right)
\end{aligned}
$$

$$
\left.\times\left(\left[\int J_{\mathrm{ss}}(\mathbf{r}, \mathbf{r}, z) \mathrm{d} \mathbf{r}+\int J_{\mathrm{pp}}(\mathbf{r}, \mathbf{r}, z) \mathrm{d} \mathbf{r}\right]^{2}\right)^{-1}\right\}^{1 / 2}
$$

This parameter remains constant upon free propagation and is widely used. But, unfortunately, the procedure used to calculate $P_{\text {st }}$ involves integration of the beam intensity over the full detection area and this only makes sense if the light field is assumed to exhibit a uniformly distributed polarization state over its cross-sectional region.

- Local and weighted degrees of polarization, $P(\mathbf{r}, z)$ and $P_{\mathrm{w}}(z)$ :

$$
\begin{aligned}
& P(\mathbf{r}, z) \\
& \quad=\sqrt{\frac{\left[J_{\mathrm{ss}}(\mathbf{r}, \mathbf{r}, z)-J_{\mathrm{pp}}(\mathbf{r}, \mathbf{r}, z)\right]^{2}+4\left|J_{\mathrm{sp}}(\mathbf{r}, \mathbf{r}, z)\right|^{2}}{\left[J_{\mathrm{ss}}(\mathbf{r}, \mathbf{r}, z)+J_{\mathrm{pp}}(\mathbf{r}, \mathbf{r}, z)\right]^{2}}}
\end{aligned}
$$

and

$$
P_{\mathrm{w}}(z)=\frac{\iint I(\mathbf{r}, z) P(\mathbf{r}, z) \mathrm{d} \mathbf{r}}{\iint I(\mathbf{r}, z) \mathrm{d} \mathbf{r}},
$$

where $I(\mathbf{r}, z)$ is the intensity at plane $z$.

The parameter $P_{\mathrm{w}}$ essentially represents the local degree of polarization averaged and normalized over those transverse regions where the intensity is significant.

- Generalized degree of polarization $P_{\mathrm{G}}$ :

$$
\begin{aligned}
& P_{\mathrm{G}}(z)=\left\{\left[\left(I_{\mathrm{s}}\left\langle r^{2}\right\rangle_{\mathrm{s}} k^{2}+I_{\mathrm{s}}\left\langle\eta^{2}\right\rangle_{\mathrm{s}}-I_{\mathrm{p}}\left\langle r^{2}\right\rangle_{\mathrm{p}} k^{2}-I_{\mathrm{p}}\left\langle\eta^{2}\right\rangle_{\mathrm{p}}\right)^{2}\right.\right. \\
& \left.\quad+4\left(I_{\mathrm{sp}}\left\langle r^{2}\right\rangle_{\mathrm{sp}} k^{2}+I_{\mathrm{sp}}\left\langle\eta^{2}\right\rangle_{\mathrm{sp}}\right)^{2}\right]\left[\left(I_{\mathrm{s}}\left\langle r^{2}\right\rangle_{\mathrm{s}} k^{2}+I_{\mathrm{s}}\left\langle\eta^{2}\right\rangle_{\mathrm{s}}\right.\right. \\
& \left.\left.\left.\quad+I_{\mathrm{p}}\left\langle r^{2}\right\rangle_{\mathrm{p}} k^{2}+I_{\mathrm{p}}\langle\boldsymbol{\eta}\rangle_{\mathrm{p}}^{2}\right)^{2}\right]^{-1}\right\}^{1 / 2} .
\end{aligned}
$$

This parameter can be understood as a second-order measure of the polarization features of the beam [15]. More specifically, for non-uniformly totally polarized beams, $P_{\mathrm{G}}$ represents a measure of the uniformity of the polarization state over the regions of the beam crosssection where the intensity is not negligible. Values of $P_{\mathrm{G}}$ close to unity mean that the beam essentially behaves as uniformly polarized, at least over the transverse region where the intensity is significant. The value of $P_{\mathrm{G}} \approx 0$ would imply, however, the lack of an overall definite polarization state over such a peak intensity area.

\section{The relation between spatial parameters and degrees of polarization of PGSM beams}

Gaussian Schell-model (GSM) sources have been extensively investigated in the scalar case (see, for example, [22]). They provide a good model for representing partially coherent sources as well as some types of multimode laser. In the scalar treatment, GSM fields are assumed to be uniformly totally polarized. The generalization of such fields to the vectorial case, that is, the recently introduced partially polarized Gaussian Schell-model (PGSM) source, is characterized by a BCP matrix whose elements read at plane $z=0$ (the waist plane)

$J_{\alpha \beta}\left(\mathbf{r}_{1}, \mathbf{r}_{2}\right)=I_{\alpha \beta} \exp \left[-\frac{r_{1}^{2}+r_{2}^{2}}{4 \sigma_{I_{\alpha \beta}}^{2}}-\frac{\left(\mathbf{r}_{1}-\mathbf{r}_{2}\right)^{2}}{2 \mu_{\alpha \beta}^{2}}\right]$

$(\alpha, \beta=\mathrm{s}, \mathrm{p})$, 
where $\sigma_{I_{\alpha \beta}}$ and $\mu_{\alpha \beta}$ and $I_{\alpha \beta}$ are positive constants. In particular, $\sigma_{I_{\alpha \alpha}}, \mu_{\alpha \alpha}$ and $I_{\alpha \alpha} \equiv I_{\alpha}, \alpha, \beta=\mathrm{s}, \mathrm{p}$, are related, respectively, to the beam size, the transverse coherence length and the beam intensity associated with each field component, and $I_{\alpha \beta}$ (also $\left.I_{\beta \alpha}\right)$ accounts for a constant phase shift between the $\mathrm{s}$ and $\mathrm{p}$ components of the field. Since such a phase could be cancelled out by means of a suitable phase plate, to simplify the equations we will set to zero the argument of $I_{\alpha \beta}$.

In the present work we are interested on the beams generated by PGSM sources that cannot be distinguished from ordinary GSM fields when polarization measurements are disregarded (i.e., when no anisotropic element is inserted in the beam path). The BCP matrix describing such fields is

$$
\begin{aligned}
& \hat{J}\left(\mathbf{r}_{1}, \mathbf{r}_{2}\right)=\exp \left(-\frac{r_{1}^{2}+r_{2}^{2}}{4 \sigma^{2}}\right) \\
& \quad \times\left(\begin{array}{cc}
I_{\mathrm{s}} \exp \left[-\frac{\left(\mathbf{r}_{1}-\mathbf{r}_{2}\right)^{2}}{2 \mu^{2}}\right] & I_{\mathrm{sp}} \exp \left[-\frac{\left(\mathbf{r}_{1}-\mathbf{r}_{2}\right)^{2}}{2 \mu_{\mathrm{sp}}^{2}}\right] \\
I_{\mathrm{sp}} \exp \left[-\frac{\left(\mathbf{r}_{1}-\mathbf{r}_{2}\right)^{2}}{2 \mu_{\mathrm{sp}}^{2}}\right] & I_{\mathrm{p}} \exp \left[-\frac{\left(\mathbf{r}_{1}-\mathbf{r}_{2}\right)^{2}}{2 \mu^{2}}\right]
\end{array}\right),
\end{aligned}
$$

together with the conditions

$$
\begin{gathered}
\mu \leqslant \mu_{\mathrm{sp}} \leqslant \mu\left(\frac{\sqrt{I_{\mathrm{s}} I_{\mathrm{p}}}}{I_{\mathrm{sp}}}\right)^{1 / 2}, \\
I_{\mathrm{sp}}^{2} \leqslant I_{\mathrm{s}} I_{\mathrm{p}},
\end{gathered}
$$

which follow from the non-negativeness property of the BCP matrix. After some calculations, we get for this class of beams

$$
\begin{gathered}
\left\langle r^{2}\right\rangle=2\left\langle r^{2}\right\rangle_{\mathrm{s}}=2\left\langle r^{2}\right\rangle_{\mathrm{s}}=2\left\langle r^{2}\right\rangle_{\mathrm{sp}}=2 \sigma^{2}, \\
\left\langle\eta^{2}\right\rangle=2\left\langle\eta^{2}\right\rangle_{\mathrm{s}}=2\left\langle\eta^{2}\right\rangle_{\mathrm{p}}=\frac{1}{2 k^{2} \sigma^{2} \chi}, \\
\left\langle\eta^{2}\right\rangle_{\mathrm{sp}}=\frac{1}{4 k^{2} \sigma^{2} \chi_{\mathrm{sp}}},
\end{gathered}
$$

where $\chi$ and $\chi_{\text {sp }}$ are given by the expressions

$$
\begin{aligned}
& \frac{1}{\chi}=4 \sigma^{2}\left(\frac{1}{4 \sigma^{2}}+\frac{1}{\mu^{2}}\right), \\
& \frac{1}{\chi_{\mathrm{sp}}}=4 \sigma^{2}\left(\frac{1}{4 \sigma^{2}}+\frac{1}{\mu_{\mathrm{sp}}^{2}}\right) .
\end{aligned}
$$

Note that equation (14) implies the condition $\chi_{\mathrm{sp}} \geqslant \chi$.

Let us now introduce a measurable parameter, $Q_{\mathrm{sp}}$, defined in the form

$$
Q_{\mathrm{sp}}=4\left\langle r^{2}\right\rangle_{\mathrm{sp}}\left\langle\eta^{2}\right\rangle_{\mathrm{sp}}
$$

Although its mathematical structure resembles the beam quality parameter $Q, Q_{\mathrm{sp}}$ involves the cross-correlation between the orthogonal s and $\mathrm{p}$ components of the field. Both spatial parameters, $Q$ and $Q_{\mathrm{sp}}$, are closely connected with the constants $\sigma, \mu$ and $\mu_{\mathrm{sp}}$ of the beam through simple expressions, namely,

$$
\begin{aligned}
Q & =\frac{1}{k^{2} \chi}, \\
Q_{\mathrm{sp}} & =\frac{1}{k^{2} \chi_{\mathrm{sp}}} .
\end{aligned}
$$

Note that $Q_{\mathrm{sp}} \leqslant Q$ because $\chi_{\mathrm{sp}} \geqslant \chi$.

Let us now focus our attention on the polarization degrees defined in section 2 . It can be shown that the standard degree of polarization may be written for this class of fields in the form

$$
P_{\mathrm{st}}=\sqrt{\left(\frac{I_{\mathrm{s}}-I_{\mathrm{p}}}{I_{\mathrm{s}}+I_{\mathrm{p}}}\right)^{2}+\frac{4 I_{\mathrm{sp}}^{2}}{\left(I_{\mathrm{s}}+I_{\mathrm{p}}\right)^{2}}} .
$$

The local degree of polarization turns out to be

$$
P(\mathbf{r}, z)=\sqrt{\left(\frac{I_{\mathrm{s}}-I_{\mathrm{p}}}{I_{\mathrm{s}}+I_{\mathrm{p}}}\right)^{2}+\frac{4 I_{\mathrm{sp}}^{2}}{\left(I_{\mathrm{s}}+I_{\mathrm{p}}\right)^{2}} \Gamma^{2}(z) \exp \left[-a(z) r^{2}\right]},
$$

where the functions $\Gamma(z)$ and $a(z)$ describe the propagation of this polarization degree and are related to the spatial parameters $Q$ and $Q_{\text {sp }}$ by the equations

$$
\begin{gathered}
\Gamma(z)=\frac{4 \sigma^{4}+z^{2} Q}{4 \sigma^{4}+z^{2} Q_{\mathrm{sp}}}, \\
a(z)=\frac{4 \sigma^{2}}{4 \sigma^{4}+z^{2} Q}[\Gamma(z)-1] .
\end{gathered}
$$

We see that $\Gamma(z)$ is an increasing function of the propagation distance $z$, and its minimum value is reached at the waist plane (in our case $z=0$ ). Furthermore, we have

$$
\Gamma(z=0)=1 \leqslant \Gamma(z) \leqslant \Gamma(z \rightarrow \infty)=\frac{Q}{Q_{\mathrm{sp}}}=\frac{\chi_{\mathrm{sp}}}{\chi} .
$$

After some algebra, we find that the generalized degree of polarization is given by

$$
P_{\mathrm{G}}(z)=\sqrt{\left(\frac{I_{\mathrm{s}}-I_{\mathrm{p}}}{I_{\mathrm{s}}+I_{\mathrm{p}}}\right)^{2}+\frac{4 I_{\mathrm{sp}}^{2}}{\left(I_{\mathrm{s}}+I_{\mathrm{p}}\right)^{2}}\left(\frac{4 k^{2} \sigma^{4}+\frac{1}{k^{2} \chi_{\mathrm{sp}}}+\frac{z^{2}}{\chi_{\mathrm{sp}}}}{4 k^{2} \sigma^{4}+\frac{1}{k^{2} \chi}+\frac{z^{2}}{\chi}}\right)^{2}} .
$$

Accordingly, $P_{\mathrm{G}}$ decreases under free propagation, and for distances $z \gg \lambda$ becomes

$$
P_{\mathrm{G}}(z)=\sqrt{\left(\frac{I_{\mathrm{s}}-I_{\mathrm{p}}}{I_{\mathrm{s}}+I_{\mathrm{p}}}\right)^{2}+\frac{4 I_{\mathrm{sp}}^{2}}{\left(I_{\mathrm{s}}+I_{\mathrm{p}}\right)^{2}} \frac{1}{\Gamma^{2}(z)}} .
$$

Closed to the waist plane $z=0$, for usual values of the beam width and the divergence, the polarization parameters $P_{\mathrm{st}}$, $P(0, z)$ and $P_{\mathrm{G}}$ are nearly identical. It can also be shown that, at every plane $z \gg \lambda$ (including the far field), the following relation applies between the local, the generalized and the standard degree of polarization:

$$
\begin{aligned}
& {\left[P_{\mathrm{G}}^{2}(z)-\left(\frac{I_{\mathrm{s}}-I_{\mathrm{p}}}{I_{\mathrm{s}}+I_{\mathrm{p}}}\right)^{2}\right]\left[P^{2}(0, z)-\left(\frac{I_{\mathrm{s}}-I_{\mathrm{p}}}{I_{\mathrm{s}}+I_{\mathrm{p}}}\right)^{2}\right]} \\
& =\left[P_{\mathrm{st}}^{2}-\left(\frac{I_{\mathrm{s}}-I_{\mathrm{p}}}{I_{\mathrm{s}}+I_{\mathrm{p}}}\right)^{2}\right]^{2} .
\end{aligned}
$$

Finally, the far-field behaviours of these polarization degrees read

$$
\begin{aligned}
& P_{\mathrm{st}}(z \rightarrow \infty)=\sqrt{\left(\frac{I_{\mathrm{s}}-I_{\mathrm{p}}}{I_{\mathrm{s}}+I_{\mathrm{p}}}\right)^{2}+\frac{4 I_{\mathrm{sp}}^{2}}{\left(I_{\mathrm{s}}+I_{\mathrm{p}}\right)^{2}},} \\
& P(\mathbf{r}, z \rightarrow \infty) \\
& =\sqrt{\left(\frac{I_{\mathrm{s}}-I_{\mathrm{p}}}{I_{\mathrm{s}}+I_{\mathrm{p}}}\right)^{2}+\frac{4 I_{\mathrm{sp}}^{2}}{\left(I_{\mathrm{s}}+I_{\mathrm{p}}\right)^{2}}\left(\frac{Q}{Q_{\mathrm{sp}}}\right)^{2} \exp \left(-r^{2} a_{\infty}\right),} \\
& P_{\mathrm{G}}(z \rightarrow \infty)=\sqrt{\left(\frac{I_{\mathrm{s}}-I_{\mathrm{p}}}{I_{\mathrm{s}}+I_{\mathrm{p}}}\right)^{2}+\frac{4 I_{\mathrm{sp}}^{2}}{\left(I_{\mathrm{s}}+I_{\mathrm{p}}\right)^{2}}\left(\frac{Q_{\mathrm{sp}}}{Q}\right)^{2}},
\end{aligned}
$$

where $a_{\infty}$ denotes the value of $a(z)$ at the far field. 


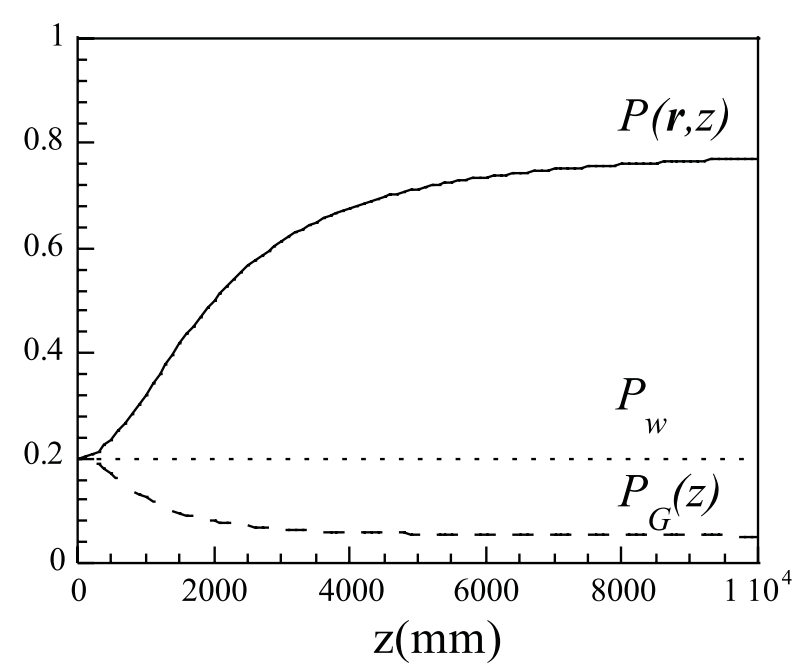

Figure 1. Degrees of polarization $P(\mathbf{r}=0, z), P_{\mathrm{G}}$ and $P_{\mathrm{w}}$ versus the propagation distance $z$ for the following values of the PGSM beam parameters: $I_{\mathrm{s}}=I_{\mathrm{p}}=0.5, I_{\mathrm{sp}}=0.1, \mu=0.1 \mathrm{~mm}, \mu_{\mathrm{sp}}=0.2 \mathrm{~mm}$, $\sigma=1 \mathrm{~mm}$ and $\lambda=633 \mathrm{~nm}$. In this example $P_{\mathrm{st}}=0.2$.

For illustrative purposes, the functions $P_{\mathrm{G}}(z), P(\mathbf{r}=0, z)$ and $P_{\mathrm{w}}$ have been plotted in figure 1 versus the propagation distance $z$ for typical values of the parameters of this class of beams.

\section{Conclusions}

The analytical relationships obtained in the above section between the spatial characteristics and the polarization behaviour of these PGSM sources enable us to infer a number of conclusions, namely:

- The local degree of polarization is a Gaussian function across the beam profile. This agrees with previous results given by Gori et al $[18,19]$.

- The local and the generalized degrees of polarization change upon free propagation according to the behaviour of function $\Gamma(z)$, which is given in terms of the measurable spatial parameters $Q$ and $Q_{\mathrm{sp}}$. This implies that, from the measurements of these parameters at the waist plane, the overall polarization structure can be determined at any plane $z$.

- The polarization degrees $P_{\mathrm{st}}, P(\mathbf{r}=0, z), P_{\mathrm{w}}$ and $P_{\mathrm{G}}(z)$ reach nearly identical values when $\mu_{\mathrm{sp}} \approx \mu$.

- When $I_{\mathrm{sp}}^{2}=I_{\mathrm{s}} I_{\mathrm{p}}$, then we have $\mu=\mu_{\mathrm{sp}}$. Consequently $\Gamma(z)=1$ and $a(z)=0$, so $P_{\mathrm{st}}=P(\mathbf{r}, z)=P_{\mathrm{G}}(z)=1$. The beam is totally and uniformly polarized at any $z$.

- When $I_{\mathrm{sp}}=0, P_{\mathrm{st}}=P(\mathbf{r}, z)=P_{\mathrm{G}}(z)=$ constant $<1$. The beam is uniformly partially polarized, and all degrees of polarization are independent of $z$. For the particular case $I_{\mathrm{s}}=I_{\mathrm{p}}$, we find that $P_{\mathrm{st}}, P(\mathbf{r}, z)$ and $P_{\mathrm{G}}(z)$ are zero. The beam is unpolarized at every plane $z$.

- When $I_{\mathrm{s}}=I_{\mathrm{p}}=I_{0}$, we have the following simple relation at any plane $z$ :

$$
P_{\mathrm{G}}(z) P(0, z)=P_{\mathrm{st}}^{2}=\frac{I_{\mathrm{sp}}^{2}}{I_{0}^{2}} .
$$

This means that the left-hand member of this equation is invariant under free propagation, and it is also independent of the coherence properties of the beam. Moreover, in this case,

$$
P_{\mathrm{w}}=P_{\mathrm{st}}=\frac{I_{\mathrm{sp}}}{I_{0}}
$$

which is independent of $z$ as well.

\section{Acknowledgments}

The research work leading to this paper was supported by the Ministerio de Ciencia y Tecnología of Spain, Project BFM2001-1356, within the framework of EUREKA projects EU-2359.

\section{References}

[1] Lavi S, Prochaska R and Keren E 1998 Generalized beam parameters and transformation law for partially coherent light Appl. Opt. 27 3696-703

[2] Simon R, Mukunda N and Sudarshan E C G 1988 Partially coherent beams and a generalized ABCD-law Opt. Commun. 65 322-8

[3] Bastiaans M J 1989 Propagation laws for the second-order moments of the Wigner distribution function in first-order optical systems Optik 82 173-81

[4] Siegman A E 1990 New developments in laser resonators Laser Resonators (Proc. SPIE vol 1224) ed D A Holmes (Bellingham, WA: SPIE Optical Engineering Press) pp 2-14

[5] Serna J, Martínez-Herrero R and Mejías P M 1991 Parametric characterization of general partially coherent beams propagating through ABCD optical systems J. Opt. Soc. Am. A 8 1094-8

[6] Weber H 1992 Propagation of higher-order intensity moments in quadratic-index media Opt. Quantum Electron. 24 1027-49

[7] Mejías P M, Weber H, Martínez-Herrero R and González-Ureña A (ed) 1993 Proc. 1st Workshop on Laser Beam Characterization (Madrid: SEDO)

[8] Weber H, Reng N, Lüdtke J and Mejías P M (ed) 1994 Proc 2nd Workshop on Laser Beam Characterization (Berlin: Festkörper-Laser-Institut Verlin)

[9] Morin M and Giesen A (ed) 1996 Proc. 3rd Int. Workshop on Laser Beam Characterization (Quebec, 1996); Proc. SPIE 2870

[10] Giesen A and Morin M (ed) 1998 Proc. 4th Int. Workshop on Laser Beam and Optics Characterization (Munich: VDI-TechnologieZentrum)

[11] Laabs $\mathrm{H}$ and Weber H (ed) 2000 Proc. 5th Int. Workshop on Laser Beam and Optics Characterization (Munich: VDI-TechnologieZentrum)

[12] Giesen A and Weber H (ed) 2002 Proc. 7th Int. Workshop on Laser Beam and Optics Characterization; Proc. SPIE 4932

[13] Lü Q, Dong S and Weber H 1995 Analysis of TEM To $_{0}$ laser beam quality degradation caused by a birefringent $\mathrm{Nd}$ :YAG rod Opt. Quantum Electron. 27 777-83

[14] Martínez-Herrero R, Mejías P M and Movilla J M 1997 Spatial characterization of partially polarized beams Opt. Lett. 22 206-8

[15] Movilla J M, Piquero G, Martínez-Herrero R and Mejías P M 1998 Parametric characterization of non-uniformly polarized beams Opt. Commun. 149 230-4

[16] Piquero G, Movilla J M, Mejías P M and Martínez-Herrero R 1999 Degree of polarization of non-uniformly partially polarized beams: a proposal $O p t$. Quantum Electron. 31 223-5

[17] Movilla J M, Piquero G, Martínez-Herrero R and Mejías P M 2000 On the measurement of the generalized degree of polarization Opt. Quantum Electron. 32 1333-42 
[18] Gori F, Santarsiero M, Piquero G, Borghi R, Mondello A and Simon R 2001 Partially polarized Gaussian Schell-model beams J. Opt. A: Pure Appl. Opt. 3 1-9

[19] Piquero G, Gori F, Romanini P, Santarsiero M, Borghi R and Mondello A 2002 Synthesis of partially polarized Gaussian Schell-model sources Opt. Coтmun. 208 9-16
[20] Gori F 1998 Matrix treatment for partially polarized, partially coherent beams Opt. Lett. 23 241-3

[21] Gori F, Santarsiero M, Vicalvi S, Borghi R and Guattari G 1998 Beam coherence-polarization matrix J. Eur. Opt. Soc. A 7 941-51

[22] Mandel L and Wolf E 1995 Optical Coherence and Quantum Optics (Cambridge: Cambridge University Press) 